\title{
Characteristics of Metabolic and Lifestyle Risk Factors in Young Japanese Patients With Coronary Heart Disease
}

\author{
A Comparison With Older Patients
}

\author{
Masako AzEgAmi, ${ }^{1}$ RN, Minoru Hongo, ${ }^{2} \mathrm{MD}$, Setsuko YANAGISAWA, ${ }^{1} \mathrm{RN}$, \\ Akie YAmAZAKI, ${ }^{1}$ RN, Kesami SAKAGUCHI, ${ }^{1}$ RM, Yoshikazu YAZAKI, ${ }^{3}$ MD, \\ and Hiroshi IMAMURA, ${ }^{4} \mathrm{MD}$
}

\section{SUMMARY}

Coronary heart disease (CHD) is recognized as a lifestyle-related disease and is the second leading cause of death in Japan. However, the cardiac risk factor profile of young patients with CHD has not been clarified in suburban areas of Japan. Our study aimed to determine metabolic and lifestyle risk factors in young patients and compare them with older patients living in suburban areas of Nagano Prefecture.

A multicenter study was conducted in 86 young (aged less than 40 years) and 91 older (aged 50 years and over) patients diagnosed with CHD from 1992 to 2002. There was a strong association between obesity and the occurrence of CHD events in young patients (odds ratio $=3.61, P=0.006$ ). Lifestyle in the young patients was characterized by a lack of physical activity and regular physical activity was found to decrease the risk of the CHD events in these patients (odds ratio $=0.31, P=0.030$ ). In older patients, hypertension was identified as an independent risk factor for CHD events.

The results of the present study have demonstrated that obesity and a lack of regular physical exercise are independent risk factors for CHD events in younger patients. Thus, the data may be useful for the effective screening of high-risk individuals and the development of educational programs for the prevention of CHD, especially in younger Japanese. (Int Heart J 2006; 47: 343-350)

Key words: Acute myocardial infarction, Angina pectoris, Young patients, Obesity, Physical activity

CORONARY heart disease (CHD) is recognized as a lifestyle-related disease and was the second leading cause of death in Japan in 2004. ${ }^{1)}$ In general, the risk of a CHD event increases with age and the number of deaths/100,000 people

From the Departments of ${ }^{1}$ Nursing, ${ }^{2}$ Cardiovascular Medicine, Shinshu University School of Health Sciences, ${ }^{3}$ Cardiovascular Medicine, and ${ }^{4}$ Emergency and Intensive Care Medicine, Shinshu University School of Medicine, Nagano, Japan.

This study was supported by a grant awarded by the Special Research Fund of the Vice-chancellor of Shinshu University 2002-2004.

Address for correspondence: Minoru Hongo, MD, Department of Cardiovascular Medicine, Shinshu University School of Health Sciences, 3-1-1 Asahi, Matsumoto, Nagano 390-8621, Japan.

Received for publication August 22, 2005.

Revised and accepted February 23, 2006. 
among Japanese males over 55 years old was 10-fold higher than in males less than 40 years old. ${ }^{2)}$ Although the risk factor profile for CHD is different between younger and older patients, ${ }^{3)}$ the profile of younger adults with CHD has not been fully examined in Japan, most likely due to limited sample size. According to epidemiological data published in the 1990s in the United States, the incidence of myocardial infarction in patients under the age of 45 years accounted for approximately $4-10 \%$ of all myocardial infarctions. ${ }^{4}$ However, more recent studies have indicated that the incidence of CHD events in men less than 40 years old was increasing. ${ }^{4,5)}$ Moreover, in our previous study that examined temporal trends in young Japanese patients with CHD over the last 20 years, we found that the number of young patients with CHD caused by multiple risk factors was increasing. ${ }^{6}$ )

A previous study has pointed out that a westernized lifestyle is strongly correlated to the increased prevalence of atherosclerotic CHD among young Japanese. ${ }^{5)}$ Although lifestyle risk factors such as diet, a lack of physical activity, and smoking may be closely related to the development of CHD in young patients, ${ }^{7}$ most studies have focused attention on only metabolic risk factors, including hypertension, dyslipidemia, diabetes, and obesity. Thus, the aims of the present study were to characterize the distribution of both metabolic and lifestyle risk factors and to analyze the contributory risk factors for CHD events in young patients living in Nagano Prefecture.

\section{Methods}

Subjects: Of the 117 general medical hospitals with over 100 beds in Nagano, 20 from various regions of the prefecture were selected and asked to participate in the study. Eligible young subjects aged less than 40 years were admitted to one of these 20 hospitals and diagnosed with CHD between 1992 and 2002. The number of young patients treated in each hospital ranged from 1 to 8 , and the total number of young patients was 91 . After excluding 3 subjects who died and 2 subjects who did not consent to participate in this study, 86 patients were enrolled (participation rate, $95 \%$ ). According to statistics published by the National Cardiovascular Center of Japan between 1977 and 1996, acute myocardial infarction (AMI) events reached a peak in men in their $60 \mathrm{~s}$ and in women in their $70 \mathrm{~s}$, and the population from 50 to 79 years accounted for over $80 \%$ of the AMI patients. ${ }^{8)}$ Therefore, older subjects who were at least 50 years old and diagnosed with CHD from 1992 to 2002 were eligible to participate in the study. Ninety-one of the 107 older patients consented to participate in the study (participation rate, 85\%).

Definition of CHD events: The definition of CHD in this study included AMI and angina pectoris. The diagnosis of AMI was defined based on symptoms, an electrocardiogram, and enzyme criteria according to the ACC/AHA guideline.9) 
Although some of the study subjects had experienced CHD events more than twice, only data from first-time CHD events were collected.

Data collection: After written informed consent was obtained, the medical records of eligible patients were reviewed and biochemical data and the presence of metabolic disorders, including obesity, hypertension, dyslipidemia, and diabetes, were noted according to the following definitions: Body mass index (BMI) was calculated as weight in kilograms divided by height in meters squared $(\mathrm{kg} /$ $\left.\mathrm{m}^{2}\right)$, and overweight $(30.0>\mathrm{BMI} \geqq 25.0)$ and obese $(\mathrm{BMI} \geqq 30.0)^{10)}$ patients were defined as obesity in this study. Hypertension was defined as systolic blood pressure $\geqq 140 \mathrm{mmHg}$ and/or diastolic pressure $\geqq 90 \mathrm{mmHg}^{11)}$ or as the use of antihypertensive medication. Dyslipidemia was defined as a history of receiving antidyslipidemia agents or as satisfying the following criteria on admission: serum total cholesterol (TC) $\geqq 220 \mathrm{mg} / \mathrm{dL}$, triglycerides $(\mathrm{TG}) \geqq 150 \mathrm{mg} / \mathrm{dL}$, highdensity lipoprotein cholesterol (HDL-C) $<40 \mathrm{mg} / \mathrm{dL}$, and/or low-density lipoprotein cholesterol (LDL-C) $\geqq 140 \mathrm{mg} / \mathrm{dL} .{ }^{12)}$ Diabetes was defined as a history of treatment with either insulin or oral hypoglycemic medication, and additional criteria were fasting plasma glucose $\geqq 126 \mathrm{mg} / \mathrm{dL}$ or casual plasma glucose $>200$ $\mathrm{mg} / \mathrm{dL} .{ }^{13)}$ To be considered eligible, data had to be measured within the first 72 hours following the CHD event. Final diagnoses, confirmed by hospital discharge records, were also taken into account. Patients were considered to have multiple risk factors when they had 3 or more metabolic risk factors, as previously described. ${ }^{6}$

Lifestyle risk factors were determined by the mailed self-administered questionnaire. Fifty-five $(64 \%)$ of the 86 younger patients and 64 (70\%) of the 91 older patients sent back the questionnaire. Participants were asked to report on their cigarette smoking, physical activity, and alcohol consumption prior to the initial hospital admission. A current smoker was defined as a person who continued smoking after the CHD event. Physical activity was considered only leisuretime activity and assessed with respect to intensity, duration, and frequency. Regular physical activity was defined as activity of moderate intensity performed for over 30 minutes at least twice a week. Habitual alcohol drinking was defined as consumption at least $20 \mathrm{~g}$ of alcoholic beverage more than 3 times a week.

All data were collected from May 2001 to December 2003.

Statistical analysis: The significance of continuous variables, such as coronary lesions, BMI, and biochemical parameters, were calculated using the 2-tailed Student $t$-test, and the differences in nominal variables were analyzed using the chisquare test or Fisher's exact 2-tailed test. Univariate logistic regression analysis was carried out to estimate the association between the onset of a CHD event and established coronary risk factors, including obesity, history of hypertension, dyslipidemia, diabetes, and smoking history, a lack of habitual physical activity, and 
habitual alcohol consumption in the younger patients. Only the significant risk factors were further analyzed using stepwise multiple logistic regression analysis to identify the independent contributory factors for CHD events in these patients. All analyses were performed with SPSS software (version 11) and a $P$ value of less than 0.05 was considered statistically significant.

\section{RESULTS}

Gender and disease classifications were significantly different between the younger and older patients. The percentage of younger female patients was significantly less than that of the older female patients. The prevalence of AMI was significantly greater and the number of coronary lesions was less in the younger patient group than in the older patient group (Table I).

In the younger patients, the prevalence of obesity was significantly increased, and therefore, the calculated BMI was significantly greater compared with the older patients. Although the difference was not statistically significant $(P$ $=0.183$ ), the younger patients tended to report awareness of being overweight earlier in life (less than 18 years) than did the older patients. The older patients had a significantly higher prevalence of hypertension than the younger patients. Serum TC, LDL cholesterol, and TG levels were significantly elevated in the younger patients compared with the older patients. In contrast, serum HDL-C level, history of dyslipidemia or diabetes, fasting plasma glucose level, and the prevalence of multiple risk factors did not differ between the 2 age groups (Table II).

The incidences of smoking history and current smoker were significantly higher in the younger group than in the older group, while regular physical activity was significantly more prevalent in the older patients. There was no difference in habitual alcohol consumption between the 2 groups (Table II).

Table I. Clinical Characteristics

\begin{tabular}{llccc}
\hline & & $\begin{array}{c}\text { Younger patients } \\
(n=86)\end{array}$ & $\begin{array}{c}\text { Older patients } \\
(n=91)\end{array}$ & $P$ value \\
\hline Age (years) & (Range) & $\begin{array}{c}34.8 \pm 4.1 \\
20-39\end{array}$ & $61.9 \pm 7.7$ & \\
\multirow{2}{*}{ Gender } & Male & $82(95 \%)$ & $74(81 \%)$ & 0.002 \\
\multirow{2}{*}{ Diagnosis of CHD } & Female & $4(5 \%)$ & $17(19 \%)$ & \multirow{2}{*}{0.001} \\
\multirow{2}{*}{ Number of coronary lesions } & AMI & $62(72 \%)$ & $44(48 \%)$ & \multirow{2}{*}{ AP } \\
& & $24(28 \%)$ & $47(52 \%)$ & \multirow{2}{*}{$1.1 \pm 0.8$} \\
\hline
\end{tabular}

Data are presented as mean $\pm \mathrm{SD}$.

$\mathrm{AMI}$ indicates acute myocardial infarction and AP, angina pectoris. 
Table II. Cardiac Risk Factor Profile

\begin{tabular}{lccccc}
\hline Risk factors & $n$ & Younger patients & $n$ & Older patients & $P$ value \\
\hline Obesity $(\mathrm{BMI} \geqq 25)$ & 75 & $43(57 \%)$ & 86 & $32(37 \%)$ & 0.011 \\
BMI $\left(\mathrm{kg} / \mathrm{m}^{2}\right)$ & 75 & $25.7 \pm 3.8$ & 85 & $24.2 \pm 3.6$ & 0.010 \\
History of hypertension & 86 & $27(31 \%)$ & 83 & $50(60 \%)$ & $<0.001$ \\
Diagnosis of FH & 85 & $9(10 \%)$ & 81 & $3(3 \%)$ & \\
History of dyslipidemia & 85 & $48(56 \%)$ & 81 & $41(51 \%)$ & $\mathrm{NS}$ \\
Serum total cholesterol (mg/dL) & 78 & $224.0 \pm 60.0$ & 79 & $198.4 \pm 41.5$ & 0.002 \\
Serum triglycerides (mg/dL) & 78 & $210.7 \pm 198.0$ & 78 & $137.7 \pm 92.0$ & 0.004 \\
Serum HDL-cholesterol (mg/dL) & 70 & $47.5 \pm 18.3$ & 53 & $48.0 \pm 13.9$ & $\mathrm{NS}$ \\
Serum LDL-cholesterol (mg/dL) & 70 & $132.9 \pm 53.9$ & 54 & $116.5 \pm 36.1$ & 0.044 \\
History of diabetes & 84 & $19(23 \%)$ & 83 & $29(35 \%)$ & $\mathrm{NS}$ \\
Fasting plasma glucose (mg/dL) & 63 & $130.5 \pm 71.0$ & 60 & $123.8 \pm 42.4$ & $\mathrm{NS}$ \\
Number of patients with MRFs & 85 & $20(24 \%)$ & 81 & $20(25 \%)$ & $\mathrm{NS}$ \\
Smoking history & 61 & $49(80 \%)$ & 88 & $56(64 \%)$ & 0.028 \\
Current smoker & & $16(26 \%)$ & & $7(8 \%)$ & 0.002 \\
Regular physical activity & 55 & $8(15 \%)$ & 67 & $20(30 \%)$ & 0.045 \\
Habitual alcohol consumption & 53 & $9(17 \%)$ & 71 & $12(17 \%)$ & $\mathrm{NS}$ \\
\hline BMI indicates body mass index; & & & & &
\end{tabular}

BMI indicates body mass index; FH, familial hypercholesterolemia; HDL, high-density lipoprotein; LDL, low-density lipoprotein and MRFs, multiple risk factors. Current smoker is defined as continued cigarette smoking after a CHD event. Regular physical activity is defined as leisure-time activity of moderate intensity performed for over 30 minutes at least twice a week. Habitual alcohol consumption is defined as consumption of at least $20 \mathrm{~g}$ of alcohol beverages more than 3 times a week.

Table III. Results of Univariate and Multivariate Logistic Regression Analysis of Risk Factors for CHD Events in Younger Patients

\begin{tabular}{|c|c|c|c|c|c|c|}
\hline & $\begin{array}{c}\text { Crude } \\
\text { Odds Ratio }\end{array}$ & $95 \% \mathrm{CI}$ & $P$ value & $\begin{array}{c}\text { Adjusted } \\
\text { Odds Ratio }\end{array}$ & $95 \% \mathrm{CI}$ & $P$ value \\
\hline AMI & 2.61 & $1.40-4.85$ & 0.003 & & & \\
\hline Multivessel disease & 0.21 & $0.10-0.11$ & $<0.001$ & & & \\
\hline Obesity (BMI $\geqq 25)$ & 2.27 & $1.20-4.27$ & 0.011 & 3.61 & $1.43-9.11$ & 0.006 \\
\hline History of hypertension & 0.30 & $0.16-0.57$ & $<0.001$ & 0.20 & $0.08-0.52$ & 0.001 \\
\hline History of dyslipidemia & 1.27 & $0.69-2.33$ & NS & & & \\
\hline History of diabetes & 0.54 & $0.28-1.08$ & NS & & & \\
\hline Smoking history & 2.33 & $1.08-5.02$ & 0.030 & 1.61 & $0.62-4.19$ & 0.329 \\
\hline Regular physical activity & 0.40 & $0.16-1.00$ & 0.049 & 0.31 & $0.11-0.89$ & 0.030 \\
\hline Habitual alcohol consumption & 0.98 & $0.38-2.54$ & NS & & & \\
\hline
\end{tabular}

Multivessel disease is defined as having 2 or 3 coronary lesions. CI indicates confidence interval.

Multivariate logistic regression analysis demonstrated a strong association between obesity and $\mathrm{CHD}$ events in the younger patients (odds ratio $=3.61, P=$ 0.006). Also, regular physical activity was found to decrease the risk of a CHD event in these patients (odds ratio $=0.31, P=0.030$ ) (Table III). Conversely, 
hypertension was an independent risk factor for a CHD event in the older patients.

\section{DiscuSsion}

The findings of the present study indicate that the important contributory factors for a CHD event in the younger patients were obesity and physical inactivity. In contrast, hypertension was found to an independent risk factor for a CHD event in the older patients.

In the present study, the prevalence of obesity in the younger patient group was significantly greater than that of the older patients $(57 \%$ versus $37 \%, P=$ $0.011)$ and much greater than that of healthy control subjects in their $20 \mathrm{~s}(18.1 \%$ in men and $7.4 \%$ in women) and in 30 s (29.3\% in men and $14.3 \%$ in women) reported by The National Nutrition Survey in Japan, 2001. ${ }^{14)}$ According to previous Japanese case-control studies, the prevalence of obesity in young male patients with CHD aged 20 to 39 years old was $15-35 \% .^{15}$ ) Thus, the younger patients in our study had a considerably higher prevalence of obesity. It is generally recognized that obesity, per se, is an independent risk factor for CHD in western countries. ${ }^{16)}$ The Pathobiological Determinants of Atherosclerosis in Youth (PDAY) study autopsied a large sample of American youth, aged 15 to 34 years, and reported a strong association between BMI and the development of atherosclerotic lesions in coronary arteries, especially in men. ${ }^{17)}$ Obesity is also associated with additional unfavorable effects, including dyslipidemia, ${ }^{18)}$ elevated systolic blood pressure, ${ }^{19)}$ increased insulin resistance, ${ }^{19)}$ and proinflammatory state, ${ }^{20,21)}$ all of which may enhance the risk of CHD.

Although the difference was not statistically significant, the younger patients in the present study tended to be obese by about age 18 or earlier. Recently, some investigators have proposed the idea that cardiovascular disease may have its origin in childhood. Japanese cohort studies revealed that the incidence of childhood obesity that continued into adulthood ranged from $32.2 \%$ to $58.5 \%$ in men and from $41.0 \%$ to $48.0 \%$ in women. ${ }^{22,23)}$ In addition, the published cohort studies which investigated obese adults for more than 10 years suggested that past obesity, especially obesity lasting over 10 years, might cause an approximately 2-fold increase in the risk of CHD. ${ }^{24}$ ) Our data show that obesity is the most important independent metabolic risk factor for a CHD event in the younger Japanese subjects. Thus, intensive community-based intervention for primary prevention of obesity should be carried out, especially in childhood.

In the present study, a lack of physical activity was identified as another independent risk factor accounting for CHD events in the younger patients and regular physical activity was found to decrease the risk of CHD events in these 
patients. The Japanese National Health Promotion Movement, known as "Health Japan 21", recommends engaging in moderate physical activity over 30 minutes at least twice a week. ${ }^{14)}$ The National Nutritional Survey in Japan found that out of 15,000 subjects queried, $29.7 \%$ of men and $27.1 \%$ of women engaged in the recommended amount of physical activity for at least a 1-year period. ${ }^{14)}$ In our study, however, only $15 \%$ of the younger patients achieved the recommended physical activity level, whereas $30 \%$ of the older patients did so. In order to prevent obesity, moderate physical activity is an important factor and is accompanied by a low risk for CHD.

Study limitations and implications: There were limitations in this study. Aging itself influences dietary habits and the extent of physical activity. Furthermore, atherosclerosis develops gradually and the CHD risk is dependent on the sum of long-term exposure to a variety of coronary risk factors. Thus, the current questionnaire data may reflect the age-related changes in lifestyle, especially in the older patients.

Further, a well-designed, nation-wide, prospective study will be required to determine whether or not (1) our findings are representative of the younger patients with CHD in both the urban and rural areas of Japan, (2) childhood obesity accounts for the increased rate of mortality and morbidity due to CHD in adulthood, and (3) direct clinical interventions at an early age can reduce CHD events in adults. In the near future, special attention should be focused on those young subjects who are more susceptible to CHD in Japan.

Conclusions: The results of the present study have demonstrated that obesity and a lack of regular physical exercise are independent risk factors for CHD events in younger patients. Thus, the data may be useful for the effective screening of highrisk individuals and the development of educational programs for the prevention of CHD, especially in younger Japanese.

\section{ACKNOWLEDGents}

We thank all of the staff of the following collaborative hospitals participating in the study for their support and valuable suggestions: Shinshu University Hospital, Nagano Red Cross Hospital, Nagano Municipal Hospital, Nagano Chuo Hospital, National Higashi Nagano Hospital, Shinonoi General Hospital, Matsushiro General Hospital, Hokushin General Hospital, National Nagano Hospital, Komoro Kousei General Hospital, Asama General Hospital, Saku General Hospital, Toyoshina Red Cross Hospital, Aizawa Hospital, Matsumoto Kyoritsu Hospital, Marunouchi Hospital, Okaya Municipal Hospital, Suwa Red Cross Hospital, Suwa Chuo Hospital, and Iida Municipal Hospital. 


\section{REFERENCES}

1. Health and Welfare Statistics Association. Journal of Health and Welfare Statistics. Tokyo: Kousaidou Publisher; 2004; 51: 46-50. (Japanese)

2. Lawlor DA, Ebrahim S, Smith GD. Role of endogenous oestrogen in aetiology of coronary heart disease: analysis of age related trends in coronary heart disease and breast cancer in England and Wales and Japan. BMJ 2002; 325: 311-2.

3. Kapoor AS. Risk assessment, stratification, and prognosis of young patients after myocardial infarction. In: Kapoor AS, Singh BN, editors. Prognosis and Risk Assessment in Cardiovascular Disease. New York: Churchill Livingstone; 1993: 199-211.

4. Choudhury L, Marsh JD. Myocardial infarction in young patients. Am J Med 1999; 107: 254-61. (Review)

5. Imakita M, Yutani C, Strong JP, et al. Second nation-wide study of atherosclerosis in infants, children and young adults in Japan. Atherosclerosis 2001; 155: 487-97.

6. Imamura H, Izawa A, Kai R, et al. Trends over the last 20 years in the clinical background of young Japanese patients with coronary artery disease. Circ J 2004; 68: 186-91.

7. Kromhout D, Menotti A, Kesteloot H, Sans S. Prevention of coronary heart disease by diet and lifestyle: evidence from prospective cross-cultural, cohort, and intervention studies. Circulation 2002; 105: 893-8. (Review)

8. Miyamoto S, Goto Y, Sumida H, et al. Risk factors and physical activity levels at the onset of acute myocardial infarction in young men. J Cardiol 2000; 36: 75-83. (Japanese)

9. Antman EM, Braunwald E. Acute myocardial infarction. In: Braunwald E, Zipes DP, Libby P, editors. Heart Disease, 6th edn. Philadelphia: WB Saunders; 2001: 1114-223.

10. World Health Organization. Obesity: Preventing and managing the global epidemic. Geneva: WHO; 2000: 613.

11. Japanese Society of Hypertension. Guideline for the management of hypertension (JSH 2004). Tokyo: Kyourinsha Publisher; 2004: 7-15. (Japanese)

12. Hata Y, Mabuchi H, Saito Y, et al. Report of the Japan Atherosclerosis Society (JAS) guideline for diagnosis and treatment of hyperlipidemia in Japanese adults. J Atheroscler Thromb 2002; 9: 1-27.

13. Kuzuya T, Nakagawa S, Satoh J, et al. Report of the Committee on the classification and diagnostic criteria of diabetes mellitus. Diabetes Res Clin Pract 2002; 55: 65-85.

14. Ministry of Health, Labour and Welfare. The National Nutrition Survey in Japan 2001. Tokyo: Daiichi Shuppan Publisher; 2003: 28-211. (Japanese)

15. Ogawa K, Numao T, lizuka M, et al. Angiographic and coronary risk factor analyses of Japanese patients with ischemic heart disease before age 40: a multicenter cooperative study. Jpn Circ J 1996; 60: 822-30.

16. Grundy SM. Obesity, metabolic syndrome, and coronary atherosclerosis. Circulation 2002; 105: 2696-8.

17. McGill HC Jr, McMahan CA, Herderick EE, et al. Obesity accelerates the progression of coronary atherosclerosis in young men. Circulation 2002; 105: 2712-8.

18. Fung TT, Rimm EB, Spiegelman D, et al. Association between dietary patterns and plasma biomarkers of obesity and cardiovascular disease risk. Am J Clin Nutr 2001; 73: 61-7.

19. Abbasi F, Brown BW Jr, Lamendola C, McLaughlin T, Reaven GM. Relationship between obesity, insulin resistance, and coronary heart disease risk. J Am Coll Cardiol 2002; 40: 937-43.

20. Wolk R, Berger P, Lennon RJ, Brilakis ES, Somers VK. Body mass index: a risk factor for unstable angina and myocardial infarction in patients with angiographically confirmed coronary artery disease. Circulation 2003; 108: 2206-11.

21. Tchernof A, Nolan A, Sites CK, Ades PA, Poehlman ET. Weight loss reduces C-reactive protein levels in obese postmenopausal women. Circulation 2002; 105: 564-9.

22. Kotani K, Nishida M, Yamashita S, et al. Two decades of annual medical examinations in Japanese obese children: do obese children grow into obese adults? Int J Obes Relat Metab Disord 1997; 21: 912-21.

23. Togashi K, Masuda H, Rankinen T, Tanaka S, Bouchard C, Kamiya H. A 12-year follow-up study of treated obese children in Japan. Int J Obes Relat Metab Disord 2002; 26: 770-7.

24. Washio M, Hayashi R; Fukuoka Heart Study Group. Past history of obesity (overweight by WHO criteria) is associated with an increased risk of nonfatal acute myocardial infarction: a case-control study in Japan. Circ J 2004; 68: 41-6. 